九州大学学術情報リポジトリ

Kyushu University Institutional Repository

\title{
On-line Knowledge Embedding for an Interactive EC-based Montage System
}

Takagi, Hideyuki

Kyushu Institute of Design : Associate Professor

Kishi, Katsuhiro

Kyushu Institute of Design

http://hdl. handle. net/2324/1670065

出版情報 : International Conference on Knowledge-Based and Intelligent Information \& Engineering Systems : Proceedings KES. 1999, pp. 280-283, 1999-09-01. IEEE

バージョン :

権利関係 : 


\title{
On-line Knowledge Embedding for an Interactive EC-based Montage System
}

\author{
Hideyuki Takagi Katsuhiro Kishi * \\ Kyushu Institute of Design \\ 4-9-1, Shiobaru, Minami-ku, Fukuoka, 815-8540 Japan \\ takagi@kyushu-id.ac.jp TEL\&FAX +81-92-553-4555
}

Keywords: interactive evolutionary computation, montage system, interface, subjective tests

\begin{abstract}
We propose an on-line knowledge embedding method for interactive evolutionary computation (EC) to reduce human fatigue. On-line knowledge embedding is a method that a user explicitly uses searching hints that the user obtained during an interactive EC operation for the next search. To evaluate this method, we developed two interactive EC-based montage systems. The first system embedded the user's intention in an EC search and the second did not. The result of subjective tests and other evaluation measurements have shown that the proposed method realized a faster convergence than ordinary interactive EC searches.
\end{abstract}

\section{INTRODUCTION}

Interactive $\mathrm{EC}$ is a technology that optimizes a system based on subjective human evaluation. It is suitable to optimize systems whose evaluation criteria are preferential or subjective, such as artistic design support for music, sound, and graphic creation. This technology has been applied to several fields: artistic design support, such as CG, music, and industrial design; engineering, such as speech processing, hearing aid fitting, database retrieval, virtual reality, and data mining; education; and entertainment [10, 11].

Although the interactive EC has been widely used, the biggest remaining problem is human fatigue. We cannot completely avoid this problem because the interactive EC requires a human operator to interact with a tireless computer to cooperatively optimize a target system. This is why human operators of the interactive EC typically end an EC optimization search at most within 10 to 20 generations. For interactive $\mathrm{EC}$ to be a practical technology, we must solve the fatigue problem.

One approach to solving the problem is to improve the interactive EC interface to achieve acceptable system outputs within a permissible allowance

${ }^{*}$ He is working for Sony Corporation now. for human fatigue. Several approaches to improve the interface have been proposed. These approaches improve the input interface to allow discrete evaluation values $[9,6]$, screen poor EC individuals using a prediction system made by learning human evaluation characteristics $[1,5]$, the display interface by displaying system outputs in a predicted order of human evaluation $[7,8]$, and so on.

In this paper, we propose and evaluate an on-line knowledge embedding method that accepts human hints or knowledge of an optimization search during an interactive EC operation. The method is based on reducing a searching space to accelerate the EC searching convergence, which is expected to lead to less human fatigue.

\section{ON-LINE KNOWLEDGE EMBEDDING}

In a conventional interactive EC, operators input their evaluation into a given system output but cannot input their intention to optimize the search. Our role, then, in the conventional interactive $\mathrm{EC}$ is passive.

If the searching space is reduced, we can generally expect to accelerate the searching convergence. When we have a priori knowledge of an interactive EC task, we are able to constrain the searching space before the start of the interactive EC search. It is useful for the search that extemporaneous searching ideas are reflected as we operate the interactive EC. For example, when our view or judgment is that a certain part of an individual will be very good for a search, we may reduce the searching space by fixing the parameter that expresses the certain part. Another concrete example of "on-line knowledge embedding" used in section 4 is to select and fix a face part image during IEC searching as soon as an operator finds the part image is quite close to what he/she looks for. Our proposed on-line knowledge embedding method provides a mechanism to reflect our searching hints or intentions during the interactive EC operation for the following EC searching generations. 


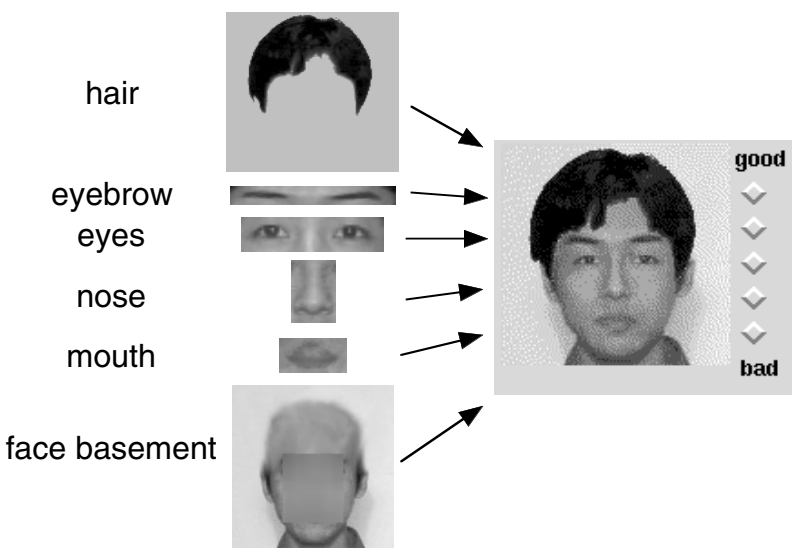

Figure 1: A face generated by combining six partimages.

This approach is good when we have a searching idea to constrain the searching space when we see or hear a phenotype of an image or sound created by the interactive EC. This means that it is only good when there is an intelligible relationship between the parameters of the searching space and their phenotype. Since the phenotype and parameters of a montage system explained in the following section have a one-to-one correspondence, the task is suitable for our proposed method. For example, in the case of an interactive EC optimizing the filter coefficients according to our evaluation of the images or sounds processed by a filter, it is difficult for the proposed method to be applied because the complexity of the mapping relationship between the filter parameters and filtered signal is difficult for a human to perceive.

We developed a montage system that has the function to embed searching hints obtained during the interactive EC operation [3]. We evaluate our proposed method using this montage system.

\section{Montage systems}

Our montage systems generate images of a face by combining six different part-images: a facial base, eyebrows, eyes, a nose, a mouth, and hair (see Figure 1.) Basically, it is similar to the well-known Caldwell \& Johnston's system [2]. Montage face image retrieval is a combinatorial optimization problem of part-images. Since our systems combine six partimages of 30 student faces, the possible number of synthesized faces is $30^{6}=729$ millions. Our task is to find a face that is similar to the given target face by combining the six parameters using the interactive EC.

$\mathrm{GA}$, one form of $\mathrm{EC}$, is used for this combinatorial optimization of images. A human operator of the interactive GA gives his or her subjective evaluation in five levels for each of the 20 faces that a GA synthesizes by combining part-images. The evaluation

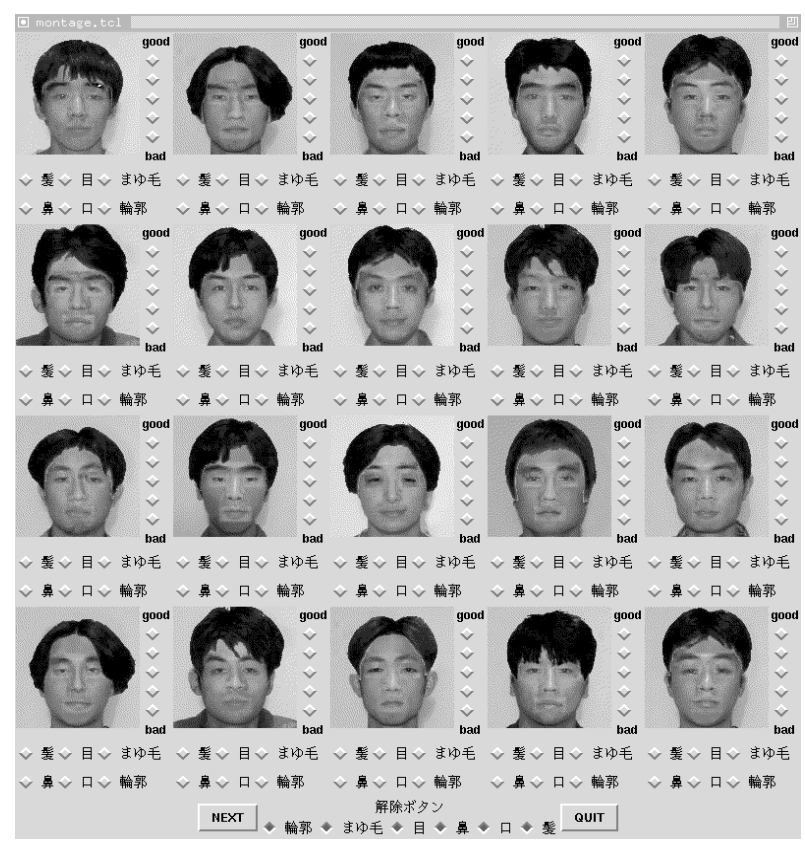

Figure 2: The user interface of a montage system. Evaluation buttons in five levels are to the right side of each face. The six buttons under each face are used to fix a part-image of a face for the following search generations. The Japanese words attached to the six buttons mean: a facial base, eyebrows, eyes, a nose, a mouth, and hair.

criterion is how the synthesized face compares to the face in the operator's memory. GA combines six new part-images according to the evaluation fitness values. These 20 generated faces are then displayed (see Figure 2.)

In section 4, we use two montage systems: our proposed on-line knowledge embedding method and a conventional one without the on-line function. The montage system with the proposed method has six buttons on each face window to embed a searching hint into the EC search as shown in Figure 3. For example, if a human operator thinks that the impression of eyes was similar to those in memory, the operator clicks the eye button. Then, the eyes of all the faces in the following search generations become the chosen eyes. The same eyes are continuously used until the release button at the bottom of the interface window in Figure 2 is clicked. From the GA point of view, it reduces the number of dimensions of searching space from six to five. This constraint contributes to accelerating the searching convergence, which is expected to reduce the human fatigue.

\section{EVAluATION EXPERIMENTS}

We evaluate the proposed on-line knowledge embedding method through subjective tests and other mea- 


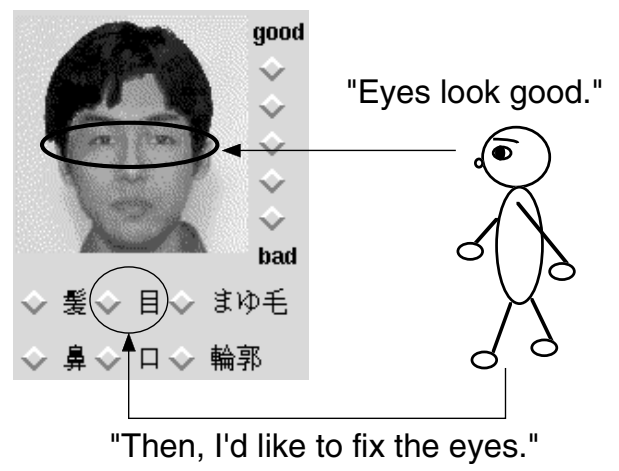

Figure 3: Buttons to fix a part-image for EC search.

surements [3, 4]. Generally, embedding hints during the interactive EC search is expected to lead to a faster conversion. At the same time, it might increase the operating time and human fatigue because of the increased number of button clicking and of the increased concentration to certain parts of a face, respectively. In this section, we evaluate the time and convergence performance of the proposed method.

\subsection{Experimental conditions}

The montage systems compared in this section are the proposed and conventional systems mentioned in section 3. Human subjects are required to generate a face that is similar to a given target face image using the two montage systems. The evaluation criterion of both systems is how the impression of a generated face compares to the target face. The operation time is also measured. The numbers of subjects, EC generations to operate, and repeated operations per system per subject in the subjective test are 14,15 , and 1 , respectively.

\subsection{Experimental evaluations}

\section{(A) subjective test \# 1}

After operating the two montage systems, 14 subjects were required to compare the best generated faces of each system and choose the better one. Table 1 shows the number of subjects who chose either better montage system. By applying the sign test, we found the difference between 12 and 2 to be statistically significant.

\section{(B) subjective test \# 2}

After operating the two systems, we asked subjects which montage system was easier to operate. Table 2 shows the number of subjects with their preferred montage system. By applying the sign test, we found the difference between 10 and 4 to be statistically insignificant.
Table 1: Subjective test result for the searching performance of our proposed on-line knowledge embedding method. The numbers of subjects who selected the face generated by each montage system as closer to the target face are listed.

\begin{tabular}{|c|c|c|}
\hline & $\begin{array}{c}\text { proposed } \\
\text { system }\end{array}$ & $\begin{array}{c}\text { conventional } \\
\text { system }\end{array}$ \\
\hline \# of subjects & 12 & 2 \\
\hline sign test & \multicolumn{2}{|c|}{ significant $(p<0.05)$} \\
\hline
\end{tabular}

Table 2: Subjective test result for the operability of our proposed on-line knowledge embedding method. The numbers of subjects who selected either montage system as easier to operate are listed.

\begin{tabular}{|c|c|c|}
\hline & $\begin{array}{c}\text { proposed } \\
\text { system }\end{array}$ & $\begin{array}{c}\text { conventional } \\
\text { system }\end{array}$ \\
\hline \# of subjects & 10 & 4 \\
\hline sign test & \multicolumn{2}{|c|}{ not significant $(p>0.05)$} \\
\hline
\end{tabular}

\section{(C) Comparison of the number of correct} part-images

Part-images of generated faces in the 5th, 10th, and 15th generations were compared with those of the target face, and the numbers that both montage systems determine as the exact part-images are counted. Although similar part-images as well as exact part-images should have higher evaluations and evaluation weight depends on the parts of a face, it is difficult to measure the similarity and the weights. Instead, we use the number of selected correct partimages as the performance index of how a similar face image is generated.

Since both systems generate 20 faces in each generation, the total number of part-images are $120=$ 6 part-images $\times 20$ faces. The average numbers of 14 subjects are shown in Table 3.

Each average value in the table comes from a set consisting of 14 numbers. We tested whether there was a significant difference between the averages of two sets and also show the results in Table 3 . The statistical test has shown that the proposed method performs better in determining the correct part-images in three test generations.

\section{(D) Comparison of operation time}

For the evaluation of operation time, we test whether there was a significant difference between the averages of two sets of the operation time taken for 15 generations. Table 4 shows the average operating time and its statistical test result.

The test result showed that operating the proposed method takes a significantly longer time per generation. 
interface research.

Table 3: The average number of part-images in a generation that are exactly same to those of the target face image. As 20 faces are displayed in each generation and each face has 6 part-images, the number in table is of 120 .

\begin{tabular}{|c|c|c|c|}
\hline \multirow{2}{*}{ counted \# of correct part-images } \\
\cline { 2 - 4 } & 5 th & 10th & 15 th \\
\hline $\begin{array}{c}\text { proposed } \\
\text { system }\end{array}$ & 12.5 & 14.1 & 17.7 \\
\hline $\begin{array}{c}\text { conventional } \\
\text { system }\end{array}$ & 4.71 & 5.64 & 6.35 \\
\hline $\begin{array}{c}\text { test for } \\
\text { the averages }\end{array}$ & $\begin{array}{c}\text { significant } \\
(p<0.05)\end{array}$ & $\begin{array}{c}\text { significant } \\
(p<0.05)\end{array}$ & $\begin{array}{c}\text { significant } \\
(p<0.01)\end{array}$ \\
\hline
\end{tabular}

Table 4: The average operation time up to 15 th generation.

\begin{tabular}{|c|c|c|}
\hline & $\begin{array}{c}\text { proposed } \\
\text { system }\end{array}$ & $\begin{array}{c}\text { conventional } \\
\text { system }\end{array}$ \\
\hline minutes & 22.9 & 16.8 \\
\hline sign test & \multicolumn{2}{|c|}{ significant $(p<0.01)$} \\
\hline
\end{tabular}

\subsection{Discussion}

From Table 4, we can say that the operation time of two systems becomes equivalent when the proposed system converges in $70 \%$ of the generations that the conventional system converges. Since Table 3 shows that the matched degree of the proposed system is three times of that of the conventional system, we might be able to say that the convergence time of the proposed system is faster than that of the conventional system.

From this discussion and the result in Table 1 , we can say that the searching performance of the proposed method is quicker and more accurate.

Regarding operability, the statistical test did not show that the difference between 10 and 4 was significant. However, these numbers lead us to expect that the difference might become significant if we increase the number of subjects.

\section{Conclusion}

We proposed the method to embed the hints of interactive EC search during operation and evaluated its performance using montage systems. Through subjective tests, comparison of the number of correct part-images and comparison of operation time, we conclude that the proposed method is faster and more accurate and may have the potential to decrease human fatigue.

We also obtained some hints on improving the interface of the interactive EC from the reports of subjects, which will be taken into account in our future

\section{REFERENCES}

[1] Biles, J. A., Anderson, P. G. and Loggi, L. W.:, "Neural network fitness functions for a musical IGA," Int'l ICSC Symposia on Intelligent Industrial Automation and Soft Computing (IIA'96/SOCO'96), pp.B39-44 (March, 1996).

[2] Caldwell, C. and Johnston, V. S. , "Tracking a criminal suspect through "face-space" with a genetic algorithm",

4th Int'l Conf. on Genetic Algorithm (ICGA'91), San Diego, CA, US, Morgan Kaufmann Publisher, pp. 416-421 (July, 1991).

[3] Kishi, K. and Takagi, H., "Developing Montage System for Study on Interactive Evolutionary Computation," SOFT 3rd Workshop on Evaluation of Heart and Mind, pp.15-18, Gamagoori, Aichi, Japan (Nov., 1998) (in Japanese).

[4] Kishi, K. and Takagi, H., "Evaluation of On-line Knowledge Embedding for Interactive Evolutionary Computation," 15th Fuzzy System Symposium, Osaka, Japan (June, 1999) (in Japanese).

[5] Nagao, M., Yamamoto, M., Suzuki, K. and Ohuchi, A., "Evaluation of the image retrieval system using interactive genetic algorithm," $J$. of Japanese Society for Artificial Intelligence, vol.13, no.5, pp.720-727 (1998) (in Japanese).

[6] Ohsaki, M., Takagi, H., and Ohya, K., "An Input Method Using Discrete Fitness Values for Interactive GA," J. of Intelligent and Fuzzy Systems, vol.6, no.1, pp.131-145 (1998).

[7] Ohsaki, M. and Takagi, H., "Reduction of the Burden of Human Interactive EC Operators - Improvement of present interface by prediction of evaluation order -," J. of Japan Artificial Intelligence Society, vol.13, no.5, pp.712-719 (1998) (in Japanese).

[8] Ohsaki, M. and Takagi, H., "Improvement of Presenting Interface by Predicting the Evaluation Order to Reduce the Burden of Human Interactive EC Operators," IEEE Int'l Conf. on System, Man, Cybernetics (SMC'98), pp.1284-1289, San Diego, CA, USA (Oct., 1998).

[9] Takagi, H. and Ohya, K., "Discrete Fitness Values for Improving the Human Interface in an Interactive GA," IEEE 3rd Int'l Conf. on Evolutionary Computation (ICEC'96), pp.109-112, Nagoya, Aichi, Japan (May, 1996).

[10] Takagi, H., "Interactive Evolutionary Computation: System Optimization Based on Human Subjective Evaluation," IEEE Int'l Conf. on Intelligent Engineering Systems (INES'98), pp.1-6, Vienna, Austria (Sept., 1998).

[11] Takagi, H., "Interactive Evolutionary Computation - Cooperation of computational intelligence and human KANSEI -," 5th Int'l Conf. on Soft Computing (IIZUKA'98), pp.41-50, World Scientific, Iizuka, Fukuoka, Japan, (Oct., 1998). 